\title{
OFF-SHELL REPRESENTATIONS WITH CENTRAL CHARGES FOR TEN-DIMENSIONAL SUPER YANG-MILLS THEORY
}

\author{
H. NICOLAI and A. VAN PROEYEN ${ }^{1}$ \\ CERN, Geneva, Switzerland
}

Received 22 March 1982

\begin{abstract}
Using Bianchi identities and commutation relations we investigate the off-shell field content of the ten-dimensional super Yang-Mills theory when central charges are introduced. Possible constraints are found, but it is not clear whether they allow the construction of an invariant action.
\end{abstract}

\section{Introduction}

The $N=4$ super Yang-Mills theory in four dimensions [1] is often compared to $N=8$ supergravity: these are the maximally extended theories if one restricts oneself to spin $\leqslant 1$ and $\leqslant 2$, respectively. Both theories are believed to share quite spectacular UV properties and might even be finite to all orders in perturbation theory [2]; for the $N=4$ theory, finiteness has been established up to three loops [3]. The investigation of these models, however, is hampered by the fact that they are known only on-shell, and it is generally assumed that only a complete off-shell formulation will completely reveal their properties. In particular one will need a superspace lagrangian in terms of unconstrained fields for the formulation of extended "superdiagrammar".

Remarkably, both theories have resisted off-shell treatment so far. As for the $N=4$ theory, it has even been plausibly argued that there exists no off-shell lagrangian possessing the full $N=4$ supersymmetry [4]. This no-go result has been corroborated by an explicit analysis of the Bianchi identities in refs. [5-7] (in ref. [7], however, a possible constraint was overlooked). This analysis was carried out for the $N=1$ theory in ten dimensions which, upon reduction, yields the $d=4, N=4$ Yang-Mills theory [1], and even though off-shell representations were found, no off-shell lagrangian has been constructed.

\footnotetext{
${ }^{1}$ Aangesteld navorser N.F.W.O., Belgium.
} 
In ref. [4] it was pointed out that the no-go result could perhaps be circumvented by introducing central charges ${ }^{\star}$. It is this possibility which we will investigate in this paper. To this purpose, we consider the following algebra in ten dimensions

$$
\left\{Q_{\alpha}, Q_{\beta}\right\}=-\frac{1}{2} \Gamma_{\alpha \beta}^{\mu} P_{\mu}-\frac{1}{2} \Gamma^{\mu_{1} \ldots \mu_{5}}{ }_{\alpha \beta} Z_{\mu_{1} \ldots \mu_{5}} .
$$

It has been shown that this is indeed the most general ten-dimensional off-shell algebra with central charges [9] if one does not enlarge the algebra by exotic objects such as fermionic central charges. Strictly speaking, the central charges which enter in (1.1) are not really central in that they do not commute with the Lorentz generators, but we will nevertheless use this terminology. In the reduction to four dimensions, one thus gets not only ordinary central charges but also tensorial ones.

As our results are somewhat inconclusive we will lay some emphasis on the methods which we employ and which combine an analysis of the Bianchi identities with explicit component calculations. Although this is largely a matter of preference, the usefulness of such a hybrid method has already been demonstrated in other contexts, and we expect it to be quite necessary for the study of algebras like (1.1) about which little is known beforehand.

In sect. 2 of this paper we construct the curvatures in a superspace with 126 extra bosonic coordinates as the group space of the algebra (1.1) and derive the Bianchi identities following general recipes [10]. One can then impose

$$
F_{\alpha \beta}=0
$$

as a conventional constraint [11] (without central charges, (1.2) would put the theory on-shell [5-7]). In sect. 3 we study the consequences of the Bianchi identities and impose two further non-conventional constraints, and in the following section we use the commutation relations to obtain more information on the higher dimensional fields and show that our additional constraints do not lead to equations of motion. Explicit component transformation rules are also given. The final section contains our conclusions.

\section{Algebra and Bianchi identities}

The superspace under consideration is spanned by the coordinates $\left(x_{\mu}, \theta_{\alpha}, z_{\mu_{1} \ldots \mu_{s}}\right)$ where $x_{\mu}$ denotes the ten-dimensional space-time variable, $\theta_{\alpha}$ a Majorana-Weyl spinor in ten dimensions and $z_{\mu_{1} \ldots \mu_{5}}$ the self-dual central charge coordinates. Thus the superspace has $10+126$ bosonic and 16 fermionic coordinates. In terms of these

* The possible relevance of central charges was also pointed out in ref. [8]. 
variables the algebra (1.1) is realized by the following differential operators ${ }^{\star}$ :

$$
\begin{gathered}
Q_{\alpha}=\frac{\partial}{\partial \theta^{\alpha}}+\frac{1}{4} \Gamma_{\mu}^{+}{ }_{\alpha}^{\beta} \theta_{\beta} \partial_{\mu}+\frac{1}{4} \Gamma_{M}^{+\beta}{ }_{\alpha} \theta_{\beta} \frac{\partial}{\partial z_{M}}, \\
P_{\mu}=\partial_{\mu}, \quad Z_{M}=\frac{\partial}{\partial z_{M}}\left(\equiv \frac{\partial}{\partial z_{\mu_{1} \ldots \mu_{3}}}\right)
\end{gathered}
$$

Covariant spinor derivatives may be straightforwardly derived in the usual fashion [10]. They are

$$
\begin{aligned}
& D_{\alpha}=i \frac{\partial}{\partial \theta^{\alpha}}-\frac{1}{4} i \Gamma_{\mu}^{+{ }_{\alpha}^{\beta}} \theta_{\beta} \partial_{\mu}-\frac{1}{4} i \Gamma_{M}{ }_{\alpha}^{+\beta} \theta_{\beta} \frac{\partial}{\partial z_{M}}, \\
& D_{\mu}=\partial_{\mu}, \quad D_{M}=\frac{\partial}{\partial z_{M}}
\end{aligned}
$$

and they satisfy

$$
\begin{aligned}
& \left\{D_{\alpha}, D_{\beta}\right\}=-\frac{1}{2} \Gamma_{\mu}^{+}{ }_{\alpha \beta} \partial_{\mu}-\frac{1}{2} \Gamma_{M}{ }_{\alpha \beta}^{+} \frac{\partial}{\partial z_{M}}, \\
& \left\{Q_{\alpha}, D_{\beta}\right\}=\text { all other (anti)commutators }=0 .
\end{aligned}
$$

The commutation relations may be succinctly re-expressed as

$$
\left[D_{A}, D_{B}\right\}=T_{A B}{ }^{C} D_{C}, \quad A, \ldots=\left\{\mu, \alpha, \mu_{1} \ldots \mu_{5}\right\},
$$

where the non-vanishing components of the torsion are given by

$$
\begin{gathered}
T_{\alpha \beta}{ }^{\mu}=-\frac{1}{2} \Gamma_{\mu}{ }^{+}{ }_{\alpha \beta}, \\
T_{\alpha \beta}{ }^{M}=-\frac{1}{2} \Gamma_{M}{ }^{+}{ }_{\alpha \beta}, \\
\text { all others }=0 .
\end{gathered}
$$

For the formulation of supersymmetric Yang-Mills theories, one must also introduce superfield Yang-Mills potentials [10].

$$
\mathbb{Q}_{A}=\mathbb{Q}_{A}^{a} T^{a}, \quad T^{a}=\text { generator of Lie group },
$$

ॠ In the following, the index $M$ will always stand for the quintuple $\left[\mu_{1} \ldots \mu_{s}\right]$. 
and the corresponding gauge-covariant derivatives

$$
\mathscr{D}_{A}=D_{A}+\mathscr{Q}_{A}
$$

The commutation relations (2.4) are modified as follows:

$$
\left[\mathscr{D}_{A}, \mathscr{D}_{B}\right\}=T_{A B}{ }^{C} \mathscr{D}_{C}+F_{A B}
$$

and from (2.8) one may read off the field strengths

$$
\begin{aligned}
& F_{\mu \nu}=\partial_{\mu} \mathbb{Q}_{\nu}+\partial_{\nu} \mathbb{Q}_{\mu}+\left[\mathbb{Q}_{\mu}, \mathbb{Q}_{\nu}\right] \\
& F_{\mu \alpha}=\partial_{\mu} \mathbb{Q}_{\alpha}-D_{\alpha} \mathbb{Q}_{\mu}+\left[\mathbb{Q}_{\mu}, \mathbb{Q}_{\alpha}\right], \\
& F_{\alpha \beta}=D_{\alpha} \mathbb{Q}_{\beta}+D_{\beta} \mathbb{Q}_{\alpha}+\left\{\mathscr{Q}_{\alpha}, \mathbb{Q}_{\beta}\right\} \\
& +\frac{1}{2} \Gamma_{\mu}^{+}{ }_{\alpha \beta} \mathbb{Q}_{\mu}+\frac{1}{2} \Gamma_{M}{ }_{\alpha \beta} \mathbb{Q}_{M}, \\
& F_{\mu M}=\partial_{\mu} \mathbb{Q}_{M}-\partial_{M} \mathbb{Q}_{\mu}+\left[\mathbb{Q}_{\mu}, \mathbb{Q}_{M}\right], \\
& F_{\alpha M}=D_{\alpha} \mathbb{Q}_{M}-\partial_{M} \mathbb{Q}_{\alpha}+\left[\mathbb{Q}_{\alpha}, \mathbb{Q}_{M}\right], \\
& F_{M N}=\partial_{M} \mathscr{Q}_{N}-\partial_{N} \mathbb{Q}_{M}+\left[\mathbb{Q}_{M}, \mathbb{Q}_{N}\right],
\end{aligned}
$$

which are graded antisymmetric, i.e., $F_{A B}=-(-)^{A B} F_{B A}$. From the Ricci identity (2.8) and the generalized Jacobi identity we obtain the Bianchi identity

$$
I_{A B C}=\left(\mathscr{D}_{A} F_{B C}-T_{A B}^{D} F_{D C}\right)[A B C\}=0
$$

where the symbol $[A B C]$ is meant to project out the graded antisymmetric part in the indices $A B C$. The components of (2.10) are

$$
\begin{gathered}
\mathscr{D}_{\alpha} F_{\beta \gamma}+\frac{1}{2} \Gamma_{\mu}^{+}{ }_{\alpha \beta} F_{\mu \gamma}+\frac{1}{2} \Gamma_{M}{ }^{+}{ }_{\alpha \beta} F_{M \gamma}+(\beta \gamma \alpha)+(\gamma \alpha \beta)=0, \\
\mathscr{D}_{\alpha} F_{\beta \mu}+\mathscr{D}_{\mu} F_{\alpha \beta}-\mathscr{D}_{\beta} F_{\mu \alpha}+\frac{1}{2} \Gamma_{\nu}^{+}{ }_{\alpha \beta} F_{\nu \mu}+\frac{1}{2} \Gamma_{M}{ }_{\alpha \beta} F_{M \mu}=0, \\
\mathscr{D}_{\alpha} F_{\beta M}+\mathscr{D}_{M} F_{\alpha \beta}-\mathscr{D}_{\beta} F_{M \alpha}+\frac{1}{2} \Gamma_{\mu}^{+}{ }_{\alpha \beta} F_{\mu M}+\frac{1}{2} \Gamma_{N}{ }^{+}{ }_{\alpha \beta} F_{N M}=0, \\
\mathscr{D}_{\alpha} F_{\mu \nu}+\mathscr{D}_{\nu} F_{\alpha \mu}+\mathscr{D}_{\mu} F_{\nu \alpha}=0, \\
\mathscr{D}_{\alpha} F_{\mu M}+\mathscr{\mathscr { D }}_{M} F_{\alpha \mu}+\mathscr{\mathscr { D }}_{\mu} F_{M \alpha}=0,
\end{gathered}
$$




$$
\begin{array}{r}
\mathscr{D}_{\alpha} F_{M N}+\mathscr{D}_{N} F_{\alpha M}+\mathscr{D}_{M} F_{N \alpha}=0, \\
\mathscr{Q}_{\mu} F_{\nu \rho}+\mathscr{D}_{\rho} F_{\mu \nu}+\mathscr{D}_{\nu} F_{\rho \mu}=0, \\
\mathscr{D}_{\mu} F_{\nu M}+\mathscr{D}_{M} F_{\mu \nu}+\mathscr{D}_{\nu} F_{M \mu}=0, \\
\mathscr{D}_{\mu} F_{M N}+\mathscr{D}_{N} F_{\mu M}+\mathscr{D}_{M} F_{N \mu}=0, \\
\mathscr{D}_{M} F_{N P}+\mathscr{D}_{P} F_{M N}+\mathscr{D}_{N} F_{P M}=0 .
\end{array}
$$

Not all of these identities are in fact independent as a consequence of "identities for identities" [12].

\section{Constraints and solution of Bianchi identities}

The set of field strengths (2.9) provides an off-shell representation for the algebra which, however, contains many superfluous fields. It can be restricted by imposing covariant constraints on the field strength which should not put the theory on-shell nor render it flat. There is one condition which one can always adopt. It is called a "conventional constraint" [11] and in our case it is

$$
F_{\alpha \beta}=0
$$

It does not imply any equations of motion but rather permits one to solve, for the gauge potentials, $Q_{\mu}$ and $Q_{M}$ in terms of $Q_{\alpha}$ via $(2.9 \mathrm{c})$. Note that without central charges, (3.1) would have been too stringent because the theory would have been put on-shell $[5,6]$. We will now analyze the Bianchi identities $(2.11 \mathrm{a}-\mathrm{c})$ assuming that (3.1) holds and discuss further constraints afterwards. A very important tool for this analysis is the use of Young tableau techniques that are described in appendix $\mathbf{A}$, as well as the correspondence between irreducible representations and certain Fierz identities first observed in ref. [13].

Identity (2.11a) involves the following irreducible components:

$$
I_{\alpha \beta \gamma}: \frac{16 \cdot 17 \cdot 18}{3 !}=144 \oplus 672 \text {. }
$$

On the other hand, the field strengths appearing in $I_{\alpha \beta \gamma}$ have the following irreducible decompositions:

$$
\begin{gathered}
F_{\mu \alpha}: 10 \otimes 16=\overline{16} \oplus 144, \\
F_{\mu_{1} \ldots \mu_{5} \alpha}: 126^{+} \otimes 16=144 \oplus 1200 \oplus 672 .
\end{gathered}
$$


This shows that identity (2.11a) relates the 144's of $F_{\mu \alpha}$ and $F_{M \alpha}$ and eliminates the 672 of $F_{M \alpha}$. Multiplying (2.11a) by $\left(\Gamma_{\mu}^{-}\right)^{\beta \alpha}$ we find

$$
\begin{aligned}
F_{\mu \alpha} & =\Gamma_{\mu}^{+\beta} W_{\beta}+W_{\mu \alpha}, \\
F_{\mu_{1} \ldots \mu_{5} \alpha} & =-\frac{1}{336} \Gamma_{\left[\mu_{1} \ldots \mu_{4} \alpha\right.}^{\beta} W_{\left.\mu_{5}\right] \beta}+\Gamma_{\left[\mu_{1} \mu_{2} \alpha\right.}{ }^{\beta} W_{\left.\mu_{3} \mu_{4} \mu_{5}\right] \beta} .
\end{aligned}
$$

Here, $W_{\alpha}(\overline{16}), W_{\mu \alpha}(144)$ and $W_{\mu \nu \rho \alpha}(1200)$ are irreducible (i.e., $\Gamma$ traceless) gaugecovariant superfields. The fact that the $\overline{16}$ and the 1200 are not restricted by the identity is mirrored by the existence of corresponding Fierz identities

$$
\begin{array}{r}
\Gamma_{\mu \alpha \beta}^{+}{ }_{\alpha \beta} \Gamma_{\gamma}^{+\delta} W_{\delta}+(\beta \gamma \alpha)+(\gamma \alpha \beta)=0, \\
\Gamma_{\mu_{1} \ldots \mu_{5} \alpha \beta}^{+} \Gamma_{\mu_{1} \mu_{2} \gamma}^{+} \delta W_{\mu_{3} \mu_{\alpha} \mu_{5} \delta}+(\beta \gamma \alpha)+(\gamma \alpha \beta)=0 .
\end{array}
$$

Identity $(2.11 b)$ involves

$$
I_{\alpha \beta \mu}: \frac{16 \cdot 17}{2} \cdot 10=\left(10 \oplus 126^{+}\right) \otimes 10=1 \oplus 45 \oplus 54 \oplus 210 \oplus 1050^{+},
$$

while the relevant field strengths contain the following irreducible pieces:

$$
\begin{gathered}
F_{\mu \nu}: 45, \\
F_{\mu_{1} \ldots \mu_{\nu} \nu}: 126^{+} \otimes 10=210 \oplus 1050^{+} .
\end{gathered}
$$

Hence, identity (2.11b) may be used to express $F_{\mu \nu}$ and $F_{M \nu}$ in terms of $\mathscr{D}_{\alpha} F_{\beta \nu}$ or, equivalently,

$$
\begin{gathered}
\mathscr{D}_{\alpha} W_{\beta}: 16 \otimes \overline{16}=1 \oplus 45 \oplus 210, \\
\mathscr{D}_{\alpha} W_{\mu \beta}: 16 \otimes 144=45 \oplus 54 \oplus 210 \oplus 945 \oplus 1050^{+} .
\end{gathered}
$$

Contracting $I_{\alpha \beta \mu}$ with $\left(\Gamma_{\rho}^{-}\right)^{\alpha \beta}$ and $\left(\Gamma_{\rho_{1} \ldots \rho_{5}}^{-}\right)^{\alpha \beta}$, respectively, we get

$$
\begin{gathered}
F_{\mu \nu}=\frac{1}{4}\left(\Gamma_{\mu \nu}^{-}\right)^{\alpha \beta} \mathscr{D}_{\alpha} W_{\beta}+\frac{1}{4}\left(\Gamma_{[\mu}^{-}\right)^{\alpha \beta} \mathscr{D}_{\alpha} W_{\nu] \beta}, \\
F_{\mu_{1} \ldots \mu_{5} \nu}=\frac{1}{480}\left\{\left(\Gamma_{\mu_{1} \ldots \mu_{5}}^{-} \Gamma_{\nu}\right)^{\alpha \beta} \mathscr{D}_{\alpha} W_{\beta}+\left(\Gamma_{\mu_{1} \ldots \mu_{5}}^{-}\right)^{\alpha \beta} \mathscr{D}_{\alpha} W_{\nu \beta}\right\} .
\end{gathered}
$$

(3.9a, b) correspond to the 45 and $210 \oplus 1050$ of identity $(2.11 \mathrm{~b})$ while the 1 and 54 
parts ( $=$ antisymmetry of $F_{\mu \nu}$ in $\mu \nu$ ) entail the constraints

$$
\begin{aligned}
\mathscr{D}_{\alpha} W^{\alpha} & =0, \\
\left(T_{(\mu}^{-}\right)^{\alpha \beta} \mathscr{D}_{\alpha} W_{\nu) \beta} & =0 .
\end{aligned}
$$

The 945 of $\mathscr{\mathscr { D }}_{\alpha} W_{\mu \beta}$ remains unrestricted.

Identity (2.11c) contains

$$
\begin{aligned}
I_{\alpha \beta \mu_{1} \ldots \mu_{\mathrm{S}}}: & \left(10 \oplus 126^{+}\right) \otimes 126^{+}=54 \oplus 210 \oplus 945 \\
& \oplus 1050^{+} \oplus 1050^{+} \oplus 2772^{+} \oplus 4125 \oplus 6930^{+} .
\end{aligned}
$$

Since of the three field strengths that enter this identity two have already been determined, there remains only

$$
F_{\mu_{1} \ldots \mu_{s} \nu_{1} \ldots \nu_{5}}: \frac{125 \cdot 126}{2}=945 \oplus 6930^{+} .
$$

This field strength may then be solved in terms of ${ }_{\alpha} F_{\beta M}$ and $F_{\mu M}$ or, equivalently,

$$
\begin{gathered}
\mathscr{D}_{\alpha} W_{\beta}, \mathscr{D}_{\alpha} W_{\mu \beta}: \operatorname{see}(3.8), \\
\mathscr{D}_{\alpha} W_{\mu \nu \rho \beta}: 16 \otimes 1200=210 \oplus 945 \oplus 1050^{+} \oplus 4125 \oplus 5940 \oplus 6930^{+} .
\end{gathered}
$$

We obtain

$$
\begin{aligned}
F_{\mu_{1} \ldots \mu_{5}, \nu_{1} \ldots \nu_{5}}= & -\frac{1}{480}\left\{\frac{1}{336}\left(\Gamma_{\mu_{1} \ldots \mu_{5}} \Gamma_{\left[\nu_{1} \ldots \nu_{4}\right.}\right)^{\alpha \beta} \mathscr{D}_{\alpha} W_{\left.\nu_{5}\right] \beta}\right. \\
& \left.-\left(\Gamma_{\mu_{1} \ldots \mu_{5}} \Gamma_{\left[\nu_{1} \nu_{2}\right.}\right)^{\alpha \beta} \mathscr{D}_{\alpha} W_{\left.\nu_{3} \nu_{4} \nu_{5}\right] \beta}\right\}_{945 \oplus 6930^{+}}
\end{aligned}
$$

from the 945 and $6930^{+}$components of (3.11). The 45's of $\mathscr{D}_{\alpha} W_{\beta}$ and $\mathscr{D}_{\alpha} W_{\mu \beta}$ and the 5940 of $\mathscr{D}_{\alpha} W_{\mu \nu \rho \beta}$ are not restricted by (3.11) as they do not enter; the 54 of $\mathscr{D}_{\alpha} W_{\mu \beta}$ has already been eliminated in (3.10b) and the $2772^{+}$of (3.11) is trivially fulfilled. The 210 and 1050 components of (3.11) relate the 210 and 1050 components of $\mathscr{Q}_{\alpha} W_{\mu \nu \rho \beta}$ to those of $\mathscr{Q}_{\alpha} W_{\beta}$ and $\mathscr{Q}_{\alpha} W_{\mu \beta}$ while the 4125 of $\mathscr{Q}_{\alpha} W_{\mu \nu \rho \beta}$ is constrained to vanish by (3.11).

This exhausts the list of independent Bianchi identities since the remaining identities follow by application of covariant derivatives. Let us now list the results found so far and discuss possible further constraints. The fundamental superfields 
are $W_{\alpha}(\overline{16}), W_{\mu \alpha}(144)$ and $W_{\mu \nu \rho \alpha}(1200)$. The above analysis has shown that their spinor derivatives have the following irreducible components

$$
\begin{aligned}
& \mathscr{D}_{\alpha} W_{\beta}: 45 \oplus 210, \\
& \mathscr{D}_{\alpha} W_{\mu \beta}: 45^{\prime} \oplus 210^{\prime} \oplus 945^{\prime} \oplus 1050^{+\prime}, \\
& \mathscr{D}_{\alpha} W_{\mu \nu \rho \beta}: 210^{\prime \prime} \oplus 945^{\prime \prime} \oplus 1050^{+\prime \prime} \oplus 5940 \oplus 6930^{+},
\end{aligned}
$$

where the primes distinguish between different representations of the same dimension. We now adopt the following additional constraints:

$$
F_{\mu M}=F_{M N}=0
$$

which do not lead to any equations of motion as will be shown in the next section. Eq. (3.15) tells us that (i) $210=210^{\prime}$ and $1050^{+\prime}=0$ [from (3.9b)]; (ii) $210^{\prime}=210^{\prime \prime}$ and $1050^{+\prime \prime}=0$ [by (3.11)] and (iii) $945^{\prime}=945^{\prime \prime}$ and $6930^{+}=0$ [from (3.14)] so the above table becomes

$$
\begin{aligned}
& \mathscr{Q}_{\alpha} W_{\beta}: 45 \oplus 210, \\
& \mathscr{Q}_{\alpha} W_{\mu \beta}: 45^{\prime} \oplus 210 \oplus 945, \\
& \mathscr{Q}_{\alpha} W_{\mu \nu \rho \beta}: 210 \oplus 945 \oplus 5940
\end{aligned}
$$

(the two independent 45's are linear combinations of the physical field strength $F_{\mu \nu}$ and an auxiliary $H_{\mu \nu}$ ). The non-conventional constraints (3.15) simplify the analysis of the remaining Bianchi identities.

Identity $(2.11 d)$ determines $\mathscr{D}_{\alpha} F_{\mu \nu}$, which has the irreducible components

$$
16 \otimes 45=16 \oplus \overline{144} \oplus 560
$$

These are determined as space-time derivatives of $W_{\alpha}$ and $W_{\mu \alpha}$.

Identity (2.11e) reads

$$
\mathscr{D}_{M} F_{\mu \alpha}=\mathscr{D}_{\mu} F_{M \alpha}
$$

and tells us that the central charge components of $W_{\alpha}$ and $W_{\mu \alpha}$ are entirely 
determined in terms of space-time derivatives of $W_{\mu \alpha}$ and $W_{\mu \nu \rho \alpha}$ :

$$
\begin{aligned}
& \mathscr{D}_{\left[\mu_{1} \ldots \mu_{5}\right]} W=\frac{1}{10} \mathscr{P}\left(-\frac{1}{336} \Gamma_{\left[\mu_{1} \ldots \mu_{4}\right.} W_{\left.\mu_{5}\right]}+\Gamma_{\left[\mu_{1} \mu_{2}\right.} W_{\left.\mu_{3} \mu_{4} \mu_{5}\right]}\right), \\
& \mathscr{D}_{\left[\mu_{1} \ldots \mu_{5}\right]} W_{\nu}=\left(\mathscr{D}_{\nu}-\frac{1}{10} \Gamma_{\nu} \mathscr{W}\right)\left(-\frac{1}{336} \Gamma_{\left[\mu_{1} \ldots \mu_{4}\right.} W_{\left.\mu_{5}\right]}+\Gamma_{\left[\mu_{1} \mu_{2}\right.} W_{\left.\mu_{3} \mu_{4} \mu_{5}\right]}\right) .
\end{aligned}
$$

Identity (2.11f) has the irreducible components

$$
\begin{aligned}
I_{M N \alpha}: \frac{126 \cdot 125}{2} \times 16= & \left(945 \oplus 6930^{+}\right) \otimes 16 \\
= & (\overline{144} \oplus 560 \oplus \overline{1200} \oplus 720+\overline{3696} \oplus 8800) \\
& \oplus(1440 \oplus 8800 \oplus 5280 \oplus 34992 \oplus 30800 \oplus 29568) .
\end{aligned}
$$

This identity relates central charge components of $W_{\mu \alpha}$ and $W_{\mu \nu \rho \alpha}$. Those of $W_{\mu \alpha}$ have been determined in identity $(2.11 \mathrm{e})$ :

$$
\begin{aligned}
\mathscr{D}_{M} W_{\mu \alpha}= & \mathscr{D}_{\mu} W_{\nu}(\overline{144} \oplus 560 \oplus 720) \\
& +\mathscr{Q}_{\mu} W_{\nu_{1} \nu_{2} \nu_{3}}(560 \oplus \overline{1200} \oplus 1440 \oplus 8800) .
\end{aligned}
$$

In principle, $\mathscr{D}_{M} W_{\mu \nu \rho \alpha}$ could have the following irreducible components:

$$
\begin{aligned}
\mathscr{D}_{M} W_{\mu \nu \rho \alpha}= & 126^{+} \otimes 1200=\overline{144} \oplus 560 \oplus \overline{1200} \oplus 1440 \\
& \oplus 720 \oplus \overline{3696} \oplus 8800 \oplus 8800 \oplus 5280 \oplus \overline{25200} \\
& \oplus 34992 \oplus 30800 \oplus 29568 .
\end{aligned}
$$

From (3.20) we find which components are determined by the identity, which reduces (3.22) to

$$
\mathscr{D}_{M} W_{\mu \nu \rho \alpha}=\operatorname{as} \mathscr{D}_{M} W_{\mu \alpha}(3.21) \oplus \overline{25200}
$$

Identity $(2.11 \mathrm{~g})$ is the usual Bianchi identity

$$
\mathbb{D}_{[\mu} F_{\nu \rho]}=0
$$

and implies that the first component of $F_{\mu \nu}$ is just the physical field strength. 
Identity $(2.11 \mathrm{~h})$ states that $F_{\mu \nu}$ has no central charge components,

$$
\mathscr{Q}_{M} F_{\mu \nu}=0
$$

This result could also be obtained by working out $\left\{\mathscr{D}_{\alpha}, \mathscr{D}_{\beta}\right\} F_{\mu \nu}$, using the result of previous Bianchi identities (2.11d) and (2.11b), and the commutator rule (2.8). This illustrates the earlier mentioned mechanism of "identities for identities". Eq. (2.11h) is not an independent identity. However, we still analyze it because it gives the result (3.25) directly without the algebra work.

Identities (2.11i) and (2.11j) are identically satisfied due to the constraints (3.15).

We recapitulate our results, while giving names to the free components. The curvatures are

$$
\begin{aligned}
& F_{\mu \nu}: 45, \\
& F_{\mu \alpha}: \overline{16}\left(W_{\alpha}\right) \oplus 144\left(W_{\mu \alpha}\right), \\
& F_{M \alpha}: 144\left(W_{\mu \alpha}\right) \oplus 1200\left(W_{\mu \nu \rho \alpha}\right) .
\end{aligned}
$$

$F_{\mu \nu}$ has only nine independent components due to (3.24). Its supersymmetry and central charge transformations are determined by

$$
\begin{aligned}
& \mathscr{D}_{\alpha} F_{\mu \nu}=\mathscr{D}_{\mu} W_{\alpha}(16 \oplus \overline{144})+\mathscr{D}_{\mu} W_{\nu \alpha}(16 \oplus \overline{144} \oplus 560), \\
& \mathscr{Q}_{M} F_{\mu \nu}=0 .
\end{aligned}
$$

$F_{\mu \nu}$ is contained in $W_{\alpha}$ and/or $W_{\mu \alpha}$ according to (3.9a). The three superfields mentioned in (3.26) are then

$$
\begin{aligned}
W_{\alpha}:\left.W_{\alpha}\right|_{\theta=0} & =\lambda, \quad \text { (the physical spinor }), \\
\mathscr{D}_{\alpha} W_{\beta} & =45\left(F_{\mu \nu}+H_{\mu \nu}^{(2)}\right) \oplus 210\left(H^{(4)}\right), \\
\mathscr{D}_{M} W_{\alpha} & =16\left(\mathscr{D}_{\mu} W_{\mu \alpha}\right) \oplus 560\left(\mathscr{D}_{\mu} W_{\nu \alpha}+\mathscr{D}_{\mu} W_{\mu \nu \rho \alpha}\right) \oplus 1440\left(\mathscr{D}_{\mu} W_{\nu \rho \sigma \alpha}\right) ; \\
W_{\mu \alpha}:\left.W_{\mu \alpha}\right|_{\theta=0}= & \chi_{\mu}, \\
\mathscr{D}_{\alpha} W_{\mu \beta}= & 45\left(H^{(2)}\right) \oplus 210\left(H^{(4)}\right) \oplus 945(K), \\
\mathscr{D}_{M} W_{\mu \beta}= & \overline{144} \oplus 560 \oplus \overline{1200} \oplus 1440 \oplus 720 \oplus 8800 \\
& \left(\mathscr{D}_{\mu} W_{\nu \alpha}+\mathscr{D}_{\mu} W_{\nu \rho \sigma \alpha}\right)
\end{aligned}
$$




$$
\begin{aligned}
W_{\mu \nu \rho \alpha}:\left.W_{\mu \nu \rho \alpha}\right|_{\theta=0} & =\tau_{\mu \nu \rho}, \\
\mathscr{Q}_{\alpha} W_{\mu \nu \rho \beta} & =210\left(H^{(4)}\right) \oplus 945(K) \oplus 5940(L), \\
\mathscr{D}_{M} W_{\mu \nu \rho \beta} & =\mathscr{D}_{M} W_{\mu \beta} \oplus \overline{25200}(\sigma) .
\end{aligned}
$$

Let us conclude this discussion by comparing the previous results with those from the analysis of Bianchi identities without central charges [1,2]. In this case $\mathcal{Q}_{M}$ is absent, so $(2.9 \mathrm{c})$ teaches us that we can only constrain the 10 irreducible component of $F_{\alpha \beta}\left(10 \oplus 126^{+}\right)$as a conventional constraint. In fact the full constraint $F_{\alpha \beta}=0$ would put the theory on-shell. The curvatures are then

$$
\begin{aligned}
& F_{\alpha \beta}: 126^{+}(\mathrm{B}), \\
& F_{\mu \nu}: 45, \\
& F_{\mu \alpha}: \overline{16}(\lambda) \oplus 144(\chi) .
\end{aligned}
$$

The supersymmetry transformations constrained by Bianchi identities are

$$
\begin{aligned}
\delta B & =144(\chi) \oplus 1200(\tau), \\
\delta F_{\mu \nu} & =\mathscr{Q}_{\mu} \lambda(16 \oplus \overline{144})+\mathscr{D}_{\mu} \chi_{\nu}(16 \oplus \overline{144} \oplus 560), \\
\delta \lambda & =45\left(F_{\mu \nu}+H_{\mu \nu}^{(2)}\right) \oplus 210\left(H^{(4)}\right), \\
\delta \chi & =45\left(H^{(2)}\right) \oplus 210\left(H^{(4)}+\mathscr{D}_{\mu} B\right) \oplus 945(K) \oplus 1050^{+}\left(\mathscr{Q}_{\mu} B\right) .
\end{aligned}
$$

One finds that (3.26) - (3.28) and (3.29) and (3.30) are quite similar. The same free fields appear. But the set with central charges does not have the $B$ field $\left(126^{+}\right)$in the dimension -1 sector. The dimension -2 sector is not yet complete in (3.30). This equation does not yet contain $\delta \tau$. This has to be determined by using the commutation relation on the fields which we already know. In the next section we will do this analysis to determine the fields up to dimension $\frac{5}{2}$.

\section{Components, commutators and constraints}

In sect. 3 we analyzed the Bianchi identities. We first imposed the conventional constraint $F_{\alpha \beta}=0$, which expresses $A_{\mu}$ and $A_{M}$ as functions of the unconstrained superfield $A_{\alpha}$. It then became clear that more constraints could be used to reduce the off-shell fields. Using all the Bianchi identities we got some information about what these constraints imply. The analysis taught us all the component fields of dimen- 
sions $\frac{3}{2}$ and 2 and revealed already a new field $\sigma(\overline{25200})$ at dimension $\frac{5}{2}$. However, several questions remain unanswered.

(1) Are the extra constraints not too strong, i.e., do they not imply the equation of motion? Therefore we should still check that $\mathscr{X} \lambda=0$ is not implied. This is an equation of dimension $\frac{5}{2}$.

(2) Are there more new fields at dimension $\frac{5}{2}$ ?

(3) Are all the previously mentioned fields necessary, or can we impose more constraints? Therefore we want the transformations of the fields of dimension 2 to see what is implied by constraining one of them to zero. Also these transformations are of dimension $\frac{5}{2}$.

Therefore we will investigate all remaining equations of dimension $\frac{5}{2}$. They are in fact contained in the Bianchi identities, but at higher $\theta$ orders. So one should act with more derivatives on these identities and then use the commutator algebra to find out what are the consequences at this dimension. In fact, this reduces to the commutator algebra of two supersymmetry derivatives (or transformations, further " $\delta$ ") on the fields of dimension $\frac{3}{2}$. This analysis will also show the usefulness of the techniques of using irreducible representations for analyzing closure equations in components. The equations are always the product of the field with the symmetric application of two transformations. Using $(16 \otimes 16)_{s}=10 \oplus 126^{+}$this means that closure equations contain the irreducible representations contained in the products with 10 and with $126^{+}$.

We start by the commutator on $\lambda$. So these equations contain

$$
\begin{aligned}
10 \otimes \overline{16} & =16 \oplus \overline{144} \quad \text { which has to be } \mathscr{D}_{\mu} \lambda \\
126^{+} \otimes \overline{16} & =16 \oplus 560 \oplus 1440 \text { has to be } \mathscr{D}_{M} \lambda \text { (eq. (3.28)) }
\end{aligned}
$$

The equations involve $\delta F_{\mu \nu}$ [eq. (3.27)] and

$$
\begin{aligned}
& \delta H^{(2)}=16 \otimes 45=16 \oplus \overline{144} \oplus 560, \\
& \delta H^{(4)}=16 \otimes 210=16 \oplus \overline{144} \oplus 560 \oplus \overline{1200} \oplus 1440 .
\end{aligned}
$$

Eq. (4.1) tells us that the content of the irreducible representation in (4.2).

1440: $\delta H^{(4)}$ must give $\mathscr{D}_{M} \lambda(1440)=\mathscr{D}_{\mu} \tau(1440)$.

1200: Unconstrained by (4.1). So $\delta H^{(4)}(\overline{1200})$ can be a new field, which we call $\xi^{(3)}$.

560: We denote $\delta H^{(4)}(560)$ by $\xi^{(2)}$. Then the equation gives $\delta H^{(2)}(560)$ which will thus contain $\mathscr{D}_{\mu} \tau+\mathscr{D}_{\mu} \chi+\xi^{(2)}$.

144: If again $\delta H^{(2)}(\overline{144})$ is denoted by $\xi^{(1)}$, we obtain here $\delta H^{(2)}(\overline{144})$ as $\mathscr{D}_{\mu} \lambda+$ $\mathscr{D}_{\mu} \chi+\xi^{(1)}$. But we can use here more information. We know that the commutator closes on-shell. On-shell means that $\mathscr{\mathscr { D }} \lambda=\mathscr{Q}_{\mu} \lambda(16)$ is zero, but $\mathscr{D}_{\mu} \lambda(\overline{144})$ is present. 
Therefore these terms close without $\delta H^{(2)}$ and $\delta H^{(4)}$. We can thus conclude that $\delta H^{(2)}$ does not contain $\mathscr{D}_{\mu} \lambda(\overline{144})$

16: There are now two equations in (4.1). They fix completely both 16's in (4.2).

The result is thus

$$
\begin{aligned}
& \delta H^{(2)}=16\left(\mathscr{D}_{\mu} \lambda+\mathscr{D}_{\mu} \chi\right) \oplus \overline{144}\left(\mathscr{D}_{\mu} x+\xi^{(1)}\right) \oplus 560\left(\mathscr{D}_{\mu} \tau+\mathscr{D}_{\mu} \chi+\xi^{(2)}\right), \\
& \delta H^{(4)}=16\left(\mathscr{D}_{\mu} \lambda+\mathscr{D}_{\mu} \chi\right) \oplus \overline{144}\left(\xi^{(1)}\right) \oplus 560\left(\xi^{(2)}\right) \oplus \overline{1200}\left(\xi^{(3)}\right) \oplus 1440\left(\mathscr{D}_{\mu} \tau\right) .
\end{aligned}
$$

The same analysis can then be done for the commutator on $\chi_{\mu}$. The equations are

$$
10 \otimes 144=16 \oplus \overline{144} \oplus 560 \oplus 720 \stackrel{?}{=} \mathscr{Q}_{\nu} \chi_{\mu},
$$

$$
\begin{aligned}
126^{+} \otimes 144 & =\overline{144} \oplus 560 \oplus \overline{1200} \oplus 1440 \oplus 720 \oplus 8800 \oplus 5280 \\
& \stackrel{?}{=} \mathscr{Q}_{M} \chi_{\mu}=\mathscr{D}_{\mu} \chi+\mathscr{D}_{\mu} \tau .
\end{aligned}
$$

In the commutator enter $\delta H^{(2)}$ and $\delta H^{(4)}$ [given in (4.3)] and $\delta K$

$$
\delta K=16 \otimes 945=\overline{144} \oplus 560 \oplus \overline{1200} \oplus 720 \oplus \overline{3696} \oplus 8800 .
$$

The first important remark here is that (4.4) has an equation for the 16 representation. $\delta K$ does not contain a 16 , and $\delta H^{(2)}$ and $\delta H^{(4)}$ are already completely fixed in terms of $\mathscr{D}_{\mu} \lambda(16)$ and $\mathscr{D}_{\mu} \chi(16)$. So this equation could imply $\mathscr{D}_{\mu} \lambda(16) \equiv \mathscr{\not} \lambda=0$ and thus put the theory on-shell. However, when we use the explicit transformation rules of $\delta \chi_{\mu}$ in $H^{(2)}$ and $H^{(4)}$, and of these last fields in $\not \partial \lambda$ [the formulae are given at the end of this section, eqs. (4.11)] we see that the equation is automatically satisfied! Therefore the theory is not put on-shell.

The result for $\delta K$ after applying all the equations of (4.4) is

$$
\begin{aligned}
\delta K= & \overline{144}\left(\mathscr{D}_{\mu} x+\xi^{(1)}\right) \oplus 560\left(\mathscr{D}_{\mu} x+\mathscr{D}_{\mu} \tau+\xi^{(2)}\right) \oplus \overline{1200}\left(\mathscr{D}_{\mu} \tau+\xi^{(3)}\right) \\
& \oplus 720\left(\mathscr{D}_{\mu} \chi\right) \oplus \overline{3696}(\phi) \oplus 8800\left(\mathscr{D}_{\mu} \tau\right) .
\end{aligned}
$$

This reveals the possible existence of another dimension $\frac{5}{2}$ field: $\phi(\overline{3696})$. Further, it is important to know if all the terms in (4.6) actually occur, as this will be needed for the investigation of possible further constraints. Indeed, as we saw in the previous paragraph, there can be cancellations from contributions of $\delta H^{(2)}$ and $\delta H^{(4)}$ in the commutator, such that some terms in (4.6) are zero. However, this is obviously not 
possible for the $\overline{1200}, 720$ and 8800 representations, which shows that these are actually present.

Finally, we consider the commutator on $\tau$. It contains

$10 \otimes 1200=560 \oplus \overline{1200} \oplus 1440 \oplus 8800$,

$126^{+} \otimes 1200=\overline{144} \oplus 560 \oplus \overline{1200} \oplus 1440 \oplus 720 \oplus \overline{3696} \oplus 8800 \oplus 8800$

$\oplus 5280 \oplus \overline{25200} \oplus 34992 \oplus 30800 \oplus 29568$.

It teaches us

$$
\begin{aligned}
\delta L= & 560\left(\mathscr{D}_{\mu} \chi+\mathscr{D}_{\mu} \tau+\xi^{(2)}\right) \oplus \overline{1200}\left(\mathscr{D}_{\mu} \theta+\xi^{(3)}\right) \\
& \oplus 1440\left(\mathscr{D}_{\mu} \tau\right) \oplus \overline{3696}(\phi) \oplus 8800\left(\mathscr{D}_{\mu} \tau\right) \oplus \\
& \oplus \overline{11088}\left(\sigma^{\prime \prime}\right) \oplus 8064\left(\sigma^{\prime}\right) \oplus 25200(\sigma),
\end{aligned}
$$

where we can only be sure that the $\phi$ and the $\sigma$ actually occur. All other terms could be absent due to cancellations. We remark in (4.8) the appearance of the field $\sigma$ [see (3.23), (3.28)] and of two other new fields $\sigma^{\prime}$ and $\sigma^{\prime \prime}$. Our final field content is thus

dimension $\frac{3}{2}: \quad \lambda(\overline{16}), \quad \chi(144), \quad \tau(1200)$,

dimension 2: $\quad F_{\mu \nu}(45$, but constrained to 9$)$,

$$
H^{(2)}(45), \quad H^{(4)}(210), \quad K(945), \quad L(5940),
$$

dimension $\frac{5}{2}: \quad \xi^{(1)}(\overline{144}), \quad \xi^{(2)}(560), \quad \xi^{(3)}(\overline{1200})$,

$$
\phi(\overline{3696}), \quad \sigma^{\prime \prime}(\overline{11088}), \quad \sigma^{\prime}(8064), \quad \sigma(\overline{25200}) .
$$

If the same analysis is done for the set without central charges, we have also the dimension -1 field $B\left(126^{+}\right)$. We find exactly the same formulae and thus the same fields as in (4.9) with the addition of $B\left(126^{+}\right.$, dim 1). The content of the transformation laws is the same, apart from the addition of $\mathscr{Q}_{\mu} B\left(210 \oplus 1050^{+}\right)$to $\delta \chi$ and $\delta \theta$.

Can we now impose more constraints? If we consider non-abelian Yang-Mills, we better not have constraints which imply the vanishing of space-time derivatives of fields. Such constraints, can, for non-abelian Yang-Mills, only imply the vanishing of the field itself. It is clear from (4.3) that $H^{(2)}$ or $H^{(4)}$ cannot be put equal to zero, as this would put the theory on-shell $\left[\mathscr{D}_{\mu} \lambda(16)=0\right]$. Therefore, (3.28) shows that $\chi$ and $\tau$ are necessary. In the remarks after (4.6) we pointed out that $\mathscr{D}_{\mu} \chi$ and $\mathscr{D}_{\mu} \tau$ 
terms do occur in $\delta K$. Therefore $K$ cannot be constrained to zero either. The only remaining possibility at dimension 2 is thus $L=0$. Therefore the $\mathscr{D}_{\mu} \chi$ and $\mathscr{Q}_{\mu} \tau$ terms in (4.8) should be absent. This is a possibility which we could not exclude by the group theoretical method. This remains to be investigated using explicit calculations. If these cancellations occur the constraint would imply

$$
L(5940)=0 \rightarrow \phi=\sigma=\sigma^{\prime}=\sigma^{\prime \prime}=0
$$

and if the $\xi^{(2)}$ or $\xi^{(3)}$ fields occur in (4.8) they are also constrained to zero. This also applies in the case without central charges. There too (4.10) is the only constraint which cannot be excluded up to dimension 2 .

We finish this section by giving the transformation rules which we needed for our explicit calculations:

$$
\begin{aligned}
\delta A_{\mu}= & \frac{1}{2} \bar{\varepsilon} \Gamma_{\mu} \lambda+\bar{\varepsilon} \chi_{\mu}, \\
\delta \lambda= & -\Gamma_{\mu \nu} \varepsilon\left(\frac{1}{4} F_{\mu \nu}+H_{\mu \nu}^{(2)}\right)+\Gamma_{\mu \nu \rho \sigma} \varepsilon H_{\mu \nu \rho \sigma}^{(4)}, \\
\delta \chi_{\mu}= & \left(\delta_{\mu \nu} \Gamma_{\rho}-\frac{1}{8} \Gamma_{\mu \nu \rho}\right) \varepsilon H_{\nu \rho}^{(2)}+3\left(\delta_{\mu \nu} \Gamma_{\rho \sigma \tau}-\frac{1}{6} \Gamma_{\mu \nu \rho \sigma \tau}\right) H_{\nu \rho \sigma \tau}^{(4)} \\
& +\Gamma_{\nu \rho \sigma} \varepsilon K_{\nu \rho \sigma}, \\
\delta H_{\mu \nu}^{(2)}= & \frac{7}{240} \bar{\varepsilon} \Gamma_{\mu \nu} \mathscr{D} \lambda-\frac{1}{360} \Gamma_{\mu \nu} \mathscr{D} \cdot \chi+\bar{\varepsilon} \Gamma_{[\mu} \xi_{\nu]}^{(1)}+\bar{\varepsilon} \xi_{\mu \nu}^{(2)} \\
& +\mathscr{D}_{\mu} \chi_{\nu} \text { and } \mathscr{D}_{\mu} \tau_{\nu \rho \sigma} \text { terms, } \\
\delta H_{\mu \nu \rho \sigma}^{(4)}= & \frac{1}{5760} \bar{\varepsilon} \Gamma_{\mu \nu \rho \sigma} \mathscr{D} \lambda-\frac{1}{6720} \bar{\varepsilon} \Gamma_{\mu \nu \rho \sigma} \mathscr{D} \cdot \chi-\frac{1}{42} \bar{\varepsilon} \Gamma_{[\mu \nu \rho} \xi_{\sigma]}^{(1)} \\
& +\frac{1}{6} \bar{\varepsilon} \Gamma_{[\mu \nu} \xi_{\rho \sigma]}^{(2)}+\bar{\varepsilon} \Gamma_{[\mu} \xi_{\nu \rho \sigma]}^{(3)}+\left(\mathscr{D}_{\mu} \chi_{\nu}+\mathscr{D}_{\mu} \tau_{\nu \rho \sigma}\right) \text { terms. }
\end{aligned}
$$

\section{Conclusions}

In this paper we have studied possible off-shell representations of the ten-dimensional super Yang-Mills theory with central charges. Let us now repeat the arguments of ref. [5] which show that within a set of reasonable assumptions there can be no off-shell lagrangian. In the preceding section we found the following fields whose 
degrees of freedom are indicated in parentheses.

$\operatorname{dim} 1: \quad A_{\mu}(9)$,

$\operatorname{dim} \frac{3}{2}: \quad \lambda(\overrightarrow{16}), \quad \chi_{\mu}(144), \quad \tau_{\mu \nu \rho}(1200)$,

$\operatorname{dim} 2: \quad H_{\mu \nu}^{(2)}(45), \quad \mathbf{H}_{\mu \nu \rho \sigma}^{(4)}(210), \quad \underset{\mu \nu}{K_{\nu \rho \sigma}(945),} \quad \underset{\mu \nu}{L_{\rho \sigma \tau \omega}}(5940)$.

The physical fields are $A_{\mu}(9)$ and $\lambda(16)$, and it is obvious in which form they will appear in the lagrangian. On the other hand, the auxiliary spinors $\chi_{\mu}$ and $\tau_{\mu \nu \rho}$ must be paired with $\operatorname{dim} \frac{5}{2}$ fields of the same irreducible type in order to drop out on the mass-shell irrespective of what other irreducible representations appear at dimension $\frac{5}{2}$. Hence those latter fields cannot enter the lagrangian, and the number of fermionic degrees of freedom is given by $16+2 \times(144+1200)=2704$. Inspection of the bosonic degrees of freedom in (5.1) shows that the numbers do not match, regardless of whether a higher constraint $L(5940)=0$ can be imposed or not. Clearly, if all representations contained in the off-shell multiplet are to appear in the lagrangian, no such lagrangian can exist, and we arrive at the same conclusion as in the case without central charges. Thus, the introduction of central charges does not improve the situation unless some better set of constraints than the one proposed in this paper is found. We stress once more that in view of the dimensional argument just given, higher order algebraic or derivative constraints will not help either.

What possibilities then are left for the formulation of off-shell $N=4$ super Yang-Mills theory? One which might work is to renounce the ten-dimensional Lorentz invariance or, equivalently, the full $N=4$ supersymmetry [14]. Another, which we find rather intriguing, could be the introduction of additional "outside" multiplets which would serve as Lagrange multipliers to eliminate the unwanted fields of dimension $\geqslant \frac{5}{2}$ : this is precisely the mechanism which allows one to go off-shell in ten-dimensional linearized supergravity [15]. It is not clear whether the conventional superspace approach is adequate for this kind of problem [16].

We are indebted to P. Howe for stimulating discussions and for his help in straightening out our conventions.

\section{Appendix A}

\section{REPRESENTATIONS AND THEIR PRODUCTS}

The $S O(10)$ representations are characterized by five numbers

$$
\lambda_{1} \geqslant \lambda_{2} \geqslant \lambda_{3} \geqslant \lambda_{4} \geqslant\left|\lambda_{5}\right|
$$


For bosonic representations these are integers which correspond to the number of boxes in the different rows of the Young tableaux. If $\lambda_{5} \neq 0$, then there are five antisymmetric Lorentz indices and the representation can be self-dual or antiselfdual $\left[(1,1,1,1,1)=126^{+},(1,1,1,1,-1)=126^{-}\right]$. For spinor representations, the $\lambda_{i}$ are half integers (the number of boxes in the row $+\frac{1}{2}$ ). $\lambda_{5}$ is positive for chiral representations, and negative for antichiral representations (see appendix B): $\left(\frac{1}{2}, \frac{1}{2}, \frac{1}{2}\right.$, $\left.\frac{1}{2}, \frac{1}{2}\right)=16,\left(\frac{1}{2}, \frac{1}{2}, \frac{1}{2}, \frac{1}{2},-\frac{1}{2}\right)=\overline{16}$. The dimensions of these representations are given

TABLE 1

Dimensions of irreducible representations in $d=10$
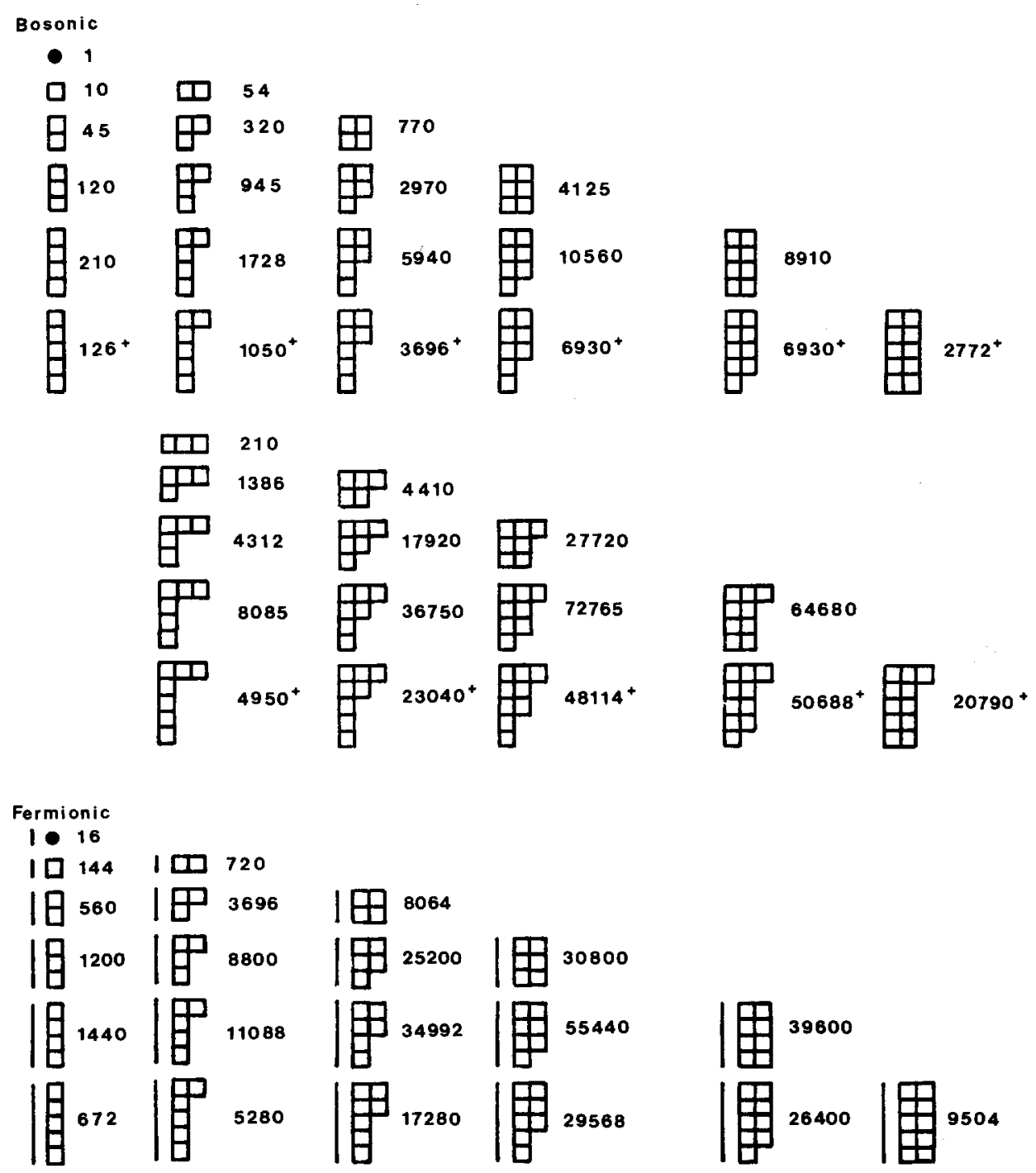
by [17]

$$
\begin{aligned}
\operatorname{dim}\left(\lambda_{1}, \lambda_{2}, \ldots, \lambda_{5}\right) & =\frac{2^{4}}{8 ! 6 ! 4 ! 2 !} \prod_{\substack{p>q \\
1}}^{5}\left(l_{p}^{2}-l_{q}^{2}\right), \\
l_{p} & =\left|\lambda_{p}\right|+5-p .
\end{aligned}
$$

Table 1 gives the dimensions for the representations with $\lambda_{1} \leqslant 3, \lambda_{2} \leqslant \frac{5}{2}$. We also note that we write the indices of the fields always in the form of a Young tableau rotated by $90^{\circ}$.

For making products of representations, one can use the methods of ref. [18]. These simplify in most of the cases we need. For example, multiplication with 10 is straightforward multiplication with $(1,0,0,0,0)+(0,0,0,0,-1)$. Note that the last one does not satisfy eq. (A.1). This is called a generalized Young tableau.

\section{Appendix B}

\section{GAMMA MATRIX ALGEBRA}

We use upper and lower spinor indices, where the charge conjugation does the raising or lowering in the following way

$$
\begin{gathered}
\bar{\varepsilon} \equiv \varepsilon^{\alpha}=c^{\alpha \beta} \varepsilon_{\beta}, \quad \varepsilon_{\alpha}=\varepsilon^{\beta} c_{\beta \alpha}, \\
c^{\gamma \alpha} c_{\gamma \beta}=\delta_{\beta}{ }^{\alpha}, \quad c^{\alpha \gamma} \Gamma_{\mu \gamma}{ }^{\delta} c_{\delta \beta}=\Gamma_{\mu \beta}{ }^{\alpha} .
\end{gathered}
$$

Normally we do not write spinor indices. It is then implied that they are contracted from upper left to lower right as in the first line of (B.1), and indices on $\Gamma$ symbols are in the same position as in the second line of (B.1).

In the appendix of ref. [5] general formulae for gamma matrix algebra in an arbitrary dimension are given. We define

$$
\Gamma_{\mu_{1} \ldots \mu_{k}}^{(k)}=\Gamma_{\left[\mu_{1}\right.} \Gamma_{\mu_{2}} \ldots \Gamma_{\left.\mu_{k}\right]}
$$

where $[\ldots]$ has weight 1 . The matrix $\Gamma^{*}$ is defined as

$$
\Gamma^{*}=\frac{i}{10 !} \varepsilon^{\mu_{1} \ldots \mu_{10}} \Gamma_{\mu_{1} \ldots \mu_{10}}
$$


It also satisfies the last equation of (B.1), and

$$
\Gamma_{\mu_{k} \ldots \mu_{1}}^{(\overleftarrow{k}} \Gamma^{*}=\frac{i}{(10-k) !} \varepsilon^{\mu_{1} \ldots \mu_{10}} \Gamma_{\mu_{k+1} \ldots \mu_{10}}^{(10-k)}
$$

$\Gamma^{(k)}$ denotes in general a gamma matrix with $k$ indices, while in $\Gamma^{(\overleftarrow{k})}$ the order of the indices is reversed (or a minus sign for $k=2,3 \bmod 4$ ). $\Gamma^{*}$ defines the chirality. A spinor which satisfies

$$
\Gamma^{*} \lambda=\lambda
$$

is chiral $(16,144, \ldots$ representation $)$. If

$$
\Gamma^{*} \lambda=-\lambda
$$

then $\lambda$ is "antichiral" $(\overline{16}, \overline{144}, \ldots)$ representation. Note that we took the supersymmetry operator as a product with 16 . Therefore the parameter $\varepsilon$ must be antichiral $(\overline{16})$. Also the superspace co-ordinate $\theta$ is antichiral. In sects. 2 and 3, $\Gamma$ matrices have been projected on chiral spinors. We use the notation

$$
\Gamma_{\mu}^{+}=\frac{1+\Gamma^{*}}{2} \Gamma_{\mu} .
$$

Duality is defined by

$$
\tilde{X}_{\mu_{1} \ldots \mu_{5}}=\frac{i}{5 !} \varepsilon_{\mu_{1} \ldots \mu_{10}} X_{\mu_{6} \ldots \mu_{10}}
$$

This satisfies

$$
\tilde{\tilde{X}}=X, \quad \tilde{X} \cdot Y=-X \cdot \tilde{Y}, \quad \Gamma^{(5)} \Gamma^{*}=\tilde{\Gamma}^{(5)} .
$$

Again, to have the $\mathbb{D}_{\left[\mu_{1} \ldots \mu_{5}\right]}$ operation to be a multiplication by $126^{+}$, we take the parameter $Z_{\left[\mu_{1} \ldots \mu_{5}\right]}$ to be antiself-dual $\left(126^{-}\right)$.

The Fierzing rule is for $d=10$

$$
M_{\alpha}^{\beta}=\frac{1}{32}\left(\sum_{n=0}^{4} \frac{2}{n !} \Gamma_{\alpha}^{(\overleftarrow{n}) \beta}\left(\Gamma_{\gamma}^{(n) \delta} M_{\delta}^{\gamma}\right)+\frac{1}{5 !} \Gamma_{\alpha}^{(5) \beta}\left(\Gamma_{\gamma}^{(5) \delta} M_{\delta}^{\gamma}\right)\right) .
$$

For gamma matrix algebra we make use of the following formulae

$$
\Gamma_{\mu_{1} \ldots \mu_{m}}^{(m)} \Gamma^{(n)} \Gamma_{\mu_{1} \ldots \mu_{m}}^{(m)}=m !(-)^{m} c(m, n) \Gamma^{(n)} .
$$

General formulae $c(m, n)$ and tables for various dimensions have been given in ref. 
[5]. These are the basis for a more general formula which we often used

$$
\begin{aligned}
\Gamma_{\rho_{1} \ldots \rho_{p} \mu_{1} \ldots \mu_{m}}^{(p+m)} \Gamma^{(n)} \Gamma_{\mu_{1} \ldots \mu_{m}}^{(m)}=(-)^{m !} m !( & x_{p}^{(m)} \Gamma_{\rho_{1} \ldots \rho_{p}} \Gamma^{(n)} \\
& +\left(\begin{array}{c}
p \\
1
\end{array}\right) x_{p}^{(m-1)} \Gamma_{\left[\rho_{1} \ldots \rho_{p-1}\right.} \Gamma^{(n)} \Gamma_{\left.\rho_{p}\right]} \\
& \left.+\left(\begin{array}{c}
p \\
2
\end{array}\right) x_{p}^{(m-2)} \Gamma_{\left[\rho_{1} \ldots \rho_{p-2}\right.} \Gamma^{(n)} \Gamma_{\left.\rho_{p-1} \rho_{p}\right]}+\cdots\right),
\end{aligned}
$$

where

$$
\begin{aligned}
(-)^{m !}= & +1 \quad \text { for } m=0,3 \bmod 4 \\
-1 & \text { for } m=1,2 \bmod 4
\end{aligned}
$$

\begin{tabular}{|c|c|c|c|}
\hline \multicolumn{4}{|c|}{$m$ odd: $x_{p}^{(m)}=0$ if $n=5 ;$ for $n=1$} \\
\hline$p$ & 1 & 3 & 5 \\
\hline 0 & 8 & 48 & 0 \\
\hline 1 & 8 & 56 & 56 \\
\hline 2 & 8 & 64 & 120 \\
\hline 3 & 8 & 72 & 192 \\
\hline 4 & 8 & 80 & 272 \\
\hline 5 & 8 & 88 & 360 \\
\hline \multicolumn{4}{|c|}{$m$ even for $\left(\begin{array}{c}n=1 \\
n=5\end{array}\right)$} \\
\hline$m$ & 0 & 2 & 4 \\
\hline 0 & 1 & $\left(\begin{array}{c}27 \\
-5\end{array}\right)$ & $\left(\begin{array}{l}42 \\
10\end{array}\right)$ \\
\hline 1 & 1 & $\left(\begin{array}{c}28 \\
-4\end{array}\right)$ & $\left(\begin{array}{r}70 \\
6\end{array}\right)$ \\
\hline 2 & 1 & $\left(\begin{array}{c}29 \\
-3\end{array}\right)$ & $\left(\begin{array}{r}99 \\
3\end{array}\right)$ \\
\hline 3 & 1 & $\left(\begin{array}{c}30 \\
-2\end{array}\right)$ & $\left(\begin{array}{r}129 \\
1\end{array}\right)$ \\
\hline 4 & 1 & $\left(\begin{array}{c}31 \\
-1\end{array}\right)$ & $\left(\begin{array}{r}160 \\
0\end{array}\right)$ \\
\hline 5 & 1 & $\left(\begin{array}{r}32 \\
0\end{array}\right)$ & $\left(\begin{array}{c}192 \\
0\end{array}\right)$ \\
\hline
\end{tabular}

TABLE 2

$x_{p}^{(m)}$ for $n=1$ and $n=5$ 
and the $x$ coefficients, whose dependence on $n$ has been suppressed, are given by

$$
\begin{array}{rlrl}
x_{p}^{(m)} & =0 & & \text { for } m<0, \\
x_{0}^{(m)} & =c(m, n) & & \text { for } m=0,3 \bmod 4 \\
& =-c(m, n) & & \text { for } m=1,2 \bmod 4, \\
x_{p}^{(m)} & =x_{p-1}^{(m)}+x_{p}^{(m-2)} . &
\end{array}
$$

The resulting coefficients are given for the most useful cases $n=1$ and $n=5$ in table 2.

\section{References}

[1] F. Gliozzi, J. Scherk and D. Olive, Nucl. Phys. B122 (1977) 256;

L. Brink, J. Schwarz and J. Scherk, Nucl. Phys. B121 (1977) 77

[2] S. Ferrara and B. Zumino, unpublished;

M. Sohnius and P. West, Phys. Lett. 100B (1981) 245;

K. Stelle, LPTENS preprint 81/24 (1981);

M. Grisaru and W. Siegel, Caltech preprint CALT-68-892 (1981)

[3] L. Avdeev, O. Tarasov and A. Vladimirov, Phys. Lett. 96B (1980) 94;

M. Grisaru, M. Roček and W. Siegel, Phys. Rev. Lett. 45 (1980) 1063;

L. Caswell and D. Zanon, Phys. Lett. 100B (1980) 152

[4] M. Roček and W. Siegel, Phys. Lett. 105B (1981) 275

[5] B. Nillson, Göteborg preprint 81-6 (1981)

[6] J. Wess, Talk at 5th Johns Hopkins Workshop on Current problems in particle theory, Baltimore (1981)

[7] R. D'Auria, P. Fré and A. da Silva, Nucl. Phys. B196 (1982) 205

[8] M. Sohnius, K. Stelle and P. West, Proc. Erice Workshop on Unification of fundamental particle interactions, ed. S. Ferrara, J. Ellis and P. van Nieuwenhuizen (Plenum, 1980)

[9] J.W. van Holten and A. Van Proeyen, CERN preprint TH-3234 (1982)

[10] J. Wess, Lectures at 8th GIFT Int. Seminar on Theoretical physics, Salamanca (1977); lecture notes in physics 77 (Springer-Verlag)

[11] J. Gates and W. Siegel, Nucl. Phys. Bl63 (1980) 519

[12] N. Dragon, Z. Phys. C2 (1979) 29;

M. Sohnius, Nucl. Phys. B136 (1978) 461

[13] R. D’Auria, P. Fré, E. Maina and T. Regge, Ann. of Phys. 139 (1982) 93

[14] P. Howe, private communication

[15] P. Howe, H. Nicolai and A. Van Proeyen, Phys. Lett. 112B (1982) 446

[16] J. Gates, Caltech preprint CALT-68-887 (1982)

[17] F. Murnaghan, The theory of group representations (Johns Hopkins Press, Baltimore, 1936) pp. 260-314

[18] G. Girardi, A. Sciarrino and P. Sorba, preprint LAPP-TH-39, CERN TH-3103 (1981), J. Phys. A, to be published; preprint LAPP-TH-45 (1981) 